\title{
Front Matter: Volume 10519
}

, "Front Matter: Volume 10519," Proc. SPIE 10519, Laser Applications in Microelectronic and Optoelectronic Manufacturing (LAMOM) XXIII, 1051901 (23 May 2018); doi: 10.1117/12.2322781

SPIE. Event: SPIE LASE, 2018, San Francisco, California, United States 


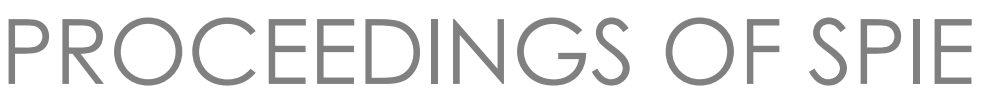

\title{
Laser Applications in Microelectronic and Optoelectronic Manufacturing (LAMOM) XXIII
}

\author{
Beat Neuenschwander \\ Costas P. Grigoropoulos \\ Tetsuya Makimura \\ Gediminas Račiukaitis \\ Editors
}

29-31 January 2018

San Francisco, California, United States

Sponsored by

SPIE

Cosponsored by

Okamoto Optics Works (Japan)

Plymouth Grating Laboratory (United States)

Published by

SPIE

Volume 10519 
The papers in this volume were part of the technical conference cited on the cover and title page. Papers were selected and subject to review by the editors and conference program committee. Some conference presentations may not be available for publication. Additional papers and presentation recordings may be available online in the SPIE Digital Library at SPIEDigitalLibrary.org.

The papers reflect the work and thoughts of the authors and are published herein as submitted. The publisher is not responsible for the validity of the information or for any outcomes resulting from reliance thereon.

Please use the following format to cite material from these proceedings:

Author(s), "Title of Paper," in Laser Applications in Microelectronic and Optoelectronic Manufacturing (LAMOM) XXIII, edited by Beat Nevenschwander, Costas P. Grigoropoulos, Tetsuya Makimura, Gediminas Račiukaitis, Proceedings of SPIE Vol. 10519 (SPIE, Bellingham, WA, 2018) Seven-digit Article CID Number.

ISSN: 0277-786X

ISSN: 1996-756X (electronic)

ISBN: 9781510615236

ISBN: 9781510615243 (electronic)

Published by

SPIE

P.O. Box 10, Bellingham, Washington 98227-0010 USA

Telephone +1 3606763290 (Pacific Time) · Fax +1 3606471445

SPIE.org

Copyright @ 2018 , Society of Photo-Optical Instrumentation Engineers.

Copying of material in this book for internal or personal use, or for the internal or personal use of specific clients, beyond the fair use provisions granted by the U.S. Copyright Law is authorized by SPIE subject to payment of copying fees. The Transactional Reporting Service base fee for this volume is $\$ 18.00$ per article (or portion thereof), which should be paid directly to the Copyright Clearance Center (CCC), 222 Rosewood Drive, Danvers, MA 01923. Payment may also be made electronically through CCC Online at copyright.com. Other copying for republication, resale, advertising or promotion, or any form of systematic or multiple reproduction of any material in this book is prohibited except with permission in writing from the publisher. The CCC fee code is 0277 $786 \mathrm{X} / 18 / \$ 18.00$.

Printed in the United States of America.

Publication of record for individual papers is online in the SPIE Digital Library.

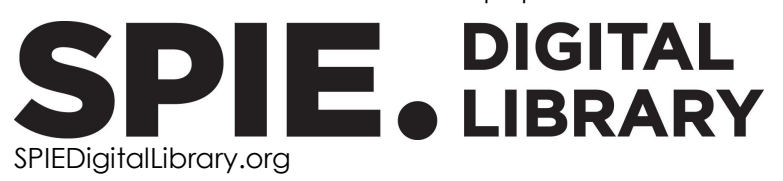

Paper Numbering: Proceedings of SPIE follow an e-First publication model. A unique citation identifier (CID) number is assigned to each article at the time of publication. Utilization of CIDs allows articles to be fully citable as soon as they are published online, and connects the same identifier to all online and print versions of the publication. SPIE uses a seven-digit CID article numbering system structured as follows:

- The first five digits correspond to the SPIE volume number.

- The last two digits indicate publication order within the volume using a Base 36 numbering system employing both numerals and letters. These two-number sets start with 00, 01, 02, 03, 04, 05, 06, 07, 08, 09, OA, OB ... 0Z, followed by 10-1Z, 20-2Z, etc. The CID Number appears on each page of the manuscript. 


\title{
Contents
}

\author{
$\checkmark \quad$ Authors \\ vii Conference Committee
}

INDUSTRIAL APPLICATIONS I: PROCESSES AND APPLICATIONS

$1051902 \quad$ Ultrafast laser ablation of copper with GHz bursts (Invited Paper) [10519-1]

1051905 Micromachining using pulse bursts: influence of the pulse duration and the number of pulses in the burst on the specific removal rate [10519-4]

$1051906 \quad$ Advanced welding of transparent materials by ultrashort laser pulses [10519-5]

INDUSTRIAL APPLICATIONS II: TOWARDS DIGITAL PHOTONICS PRODUCTION

1051907 Digital photonic production along the lines of industry 4.0 (Invited Paper) [10519-6]

1051908 Beam delivery methods for highly efficient USP-laser micro structuring of large cylindrical surfaces for printing and embossing [10519-7]

1051909 Correlation between ablation efficiency, surface morphology, and multipass capability using a 100-W 10-MHz ultrafast laser [10519-8]

10519 0A Ultra-short pulse fiber beam delivery in micromachining applications [10519-9]

DIAGNOSTICS AND ITS APPLICATIONS

10519 OB Development of a tunable high repetition rate XUV source for time-resolved photoemission studies of ultrafast dynamics at surfaces (Invited Paper) [10519-10]

10519 OC Advanced in-situ diagnostics of ultra short pulsed micromachining in glass [10519-11]

THIN FILM AND WAFER PROCESSING

$105190 \mathrm{E} \quad$ Electrical micro-heating structures on glass created by laser ablation [10519-15]

$10519 \mathrm{OH} \quad$ Tunable laser diode using partially intermixed InGaAsP multiple quantum well [10519-16] 


\section{LASER DIRECT WRITING I}

10519 OK Femtosecond written buried waveguides in silicon [10519-19]

$10519 \mathrm{OL} \quad$ Controlling properties of few-layer MoS 2 with a multi-laser process scheme [10519-20]

FUNDAMENTAL ASPECTS OF LASER-MATERIALS INTERACTION

10519 OP Relaxation processes in laser-excited dielectrics (Invited Paper) [10519-24]

10519 OT Electron dynamics in silver after ultrafast laser-excitation (Best Student Paper Award) [10519-28]

\section{LASER-INDUCED MODIFICATION OF GLASSES OR TRANSPARENT MATERIALS}

10519 oY Laser processing transparent materials with nanosecond, picosecond and femtosecond pulses for industrial applications [10519-33]

\section{LASER NANOSCALE MATERIALS PROCESSING AND MANUFACTURING}

1051915 Fabrication of hollow microneedles by optical vortex illumination [10519-40]

1051918 SERS microchips integrated with 2D periodic Cu-Ag SERS nanostructure inside a 3D microfluidics by all-femtosecond-laser-processing [10519-43]

\section{POSTER SESSION}

1051919 Microfabrication of UV transparent fluoric polymer using a conventional pulsed green laser [10519-44]

$105191 \mathrm{~A} \quad$ Femtosecond laser induced periodic nanostructures formation on medical polymer plate surface for control of cell spreading (Best Student Poster Award) [10519-47]

10519 1B Femtosecond laser-written double line waveguides in germanate and tellurite glasses [10519-48]

10519 1D Simultaneous measurement of surface profile and thickness variation of transparent parallel plate using wavelength tuning Fizeau interferometer [10519-51] 


\title{
Authors
}

Numbers in the index correspond to the last two digits of the seven-digit citation identifier (CID) article numbering system used in Proceedings of SPIE. The first five digits reflect the volume number. Base 36 numbering is employed for the last two digits and indicates the order of articles within the volume. Numbers start with 00, 01, 02, 03, 04, 05, 06, 07, 08, 09, OA, OB...0Z, followed by 10-12, 20-2Z, etc.

\author{
Allison, Thomas K., OB \\ Arndt-Staufenbiel, N., OG \\ Asai, S., 1 A \\ Audouard, Eric, 09 \\ Bai, Shi, 18 \\ Böttger, G., OG \\ Brouwer, Nils, OP \\ Bruneel, D., 05 \\ Bruening, Stephan, 08 \\ Cangueiro, L., 05 \\ Chen, Wei, OY \\ Corder, Christopher, OB \\ de Rossi, Wagner, 1B \\ Eibl, Florian, 07 \\ Eilzer, Sebastian, OA \\ Finger, J., 07 \\ Flamm, D., OC \\ Flemmer, John, 07 \\ Funck, Max C., OA \\ Gasser, Andres, 07 \\ Grossmann, D., 06, 0C \\ Gruber, A., 06 \\ Hairaye, C., 05 \\ Hanada, Y., 19 \\ Helvajian, $\mathrm{H}_{\text {., }} \mathrm{OL}$ \\ Hennig, Guido, 08 \\ Hinke, Christian, 07 \\ $\mathrm{Hu}$, Anming, 18 \\ Jäggi, Beat, 05, 08 \\ Jenne, M., 06, OC \\ Kämmer, H., OK \\ Kassab, Luciana R. P., 1B \\ Kershis, Matthew D., OB \\ Kim, Yangjin, 1D \\ Kleiner, J., OC \\ Kleinert, Jan, 02 \\ Kling, Rainer, 09 \\ Kumkar, M., 06, 0C \\ Lammers, K. A., OK \\ Li, Xinlong, OB \\ LiKamWa, Patrick, $\mathrm{OH}$ \\ Lin, Zhibin, 02 \\ Lince, J. R., OL \\ Lopez, John, 09 \\ Matsumoto, Hisashi, 02 \\ Matthäus, G., OK \\ Meiners, Wilhelm, 07 \\ Mincuzzi, Girolamo, 09 \\ Mishchik, Konstantin, 09
}

\author{
Mitsuishi, Mamoru, ID \\ Miyaji, Kunio, 15 \\ Mottay, Eric, 09 \\ Murai, K., IA \\ Neitz, M., OG \\ Nevenschwander, Beat, 05, 08 \\ Nolte, S., OK \\ Oikawa, Yoichi, 15 \\ Omatsu, Takashige, 15 \\ Ooga, T., lA \\ Ou, Zhilong, OY \\ Payton, A., OL \\ Poprawe, Reinhart, 07 \\ Queisser, M., OG \\ Ramos de Campos, J. A., 05 \\ Reininghaus, M., 07 \\ Rethfeld, Baerbel, OP, OT \\ S. da Silva, Diego, 1B \\ Samad, Ricardo E., 1B \\ Sato, Y., IA \\ Schleifenbaum, Johannes Henrich, 07 \\ Schneider-Ramelow, M., OG \\ Schopphoven, Thomas, 07 \\ Stirnimann, Adrian, 08 \\ Sugioka, Koji, 18 \\ Sugita, Naohiko, 1D \\ Tabbakh, Thamer, $\mathrm{OH}$ \\ Takenaka, K., IA \\ Takeuchi, M., 19 \\ Tao, Sha, OY \\ Toyoda, Kohei, 15 \\ Tsukamoto, M., $1 \mathrm{~A}$ \\ Voshage, Maximilian, 07 \\ Wang, Brian, OY \\ Wang, Dexiang, 15 \\ Watanabe, W., OK \\ Weber, Sebastian T., OT \\ Wedel, Björn, OA \\ Weingarten, Christian, 07 \\ Wetter, Niklaus U., 1B \\ White, Michael G., OB \\ Willenborg, Edgar, 07 \\ Wuenschell, J. K., OL \\ Zarei, Omid, 07 \\ Zhang, Jie, OY \\ Zhao, Jay, OY \\ Zhao, Peng, OB \\ Ziegler, Stephan, 07 \\ Zimmermann, F., 06, 0C
}


Proc. of SPIE Vol. $105191051901-6$

Downloaded From: https://www.spiedigitallibrary.org/conference-proceedings-of-spie on 26 Apr 2023 Terms of Use: https://www.spiedigitallibrary.org/terms-of-use 


\section{Conference Committee}

Symposium Chairs

Koji Sugioka, RIKEN (Japan)

Reinhart Poprawe, Fraunhofer-Institut für Lasertechnik (Germany)

Symposium Co-chairs

Xianfan Xu, Purdue University (United States)

Beat Nevenschwander, Berner Fachhochschule Technik und Informatik (Switzerland)

Program Track Chairs

Henry Helvajian, The Aerospace Corporation (United States)

Guido Hennig, Daetwyler Graphics AG (Switzerland)

Conference Chairs

Beat Neuenschwander, Berner Fachhochschule Technik und Informatik (Switzerland)

Costas P. Grigoropoulos, University of California, Berkeley

(United States)

Tetsuya Makimura, University of Tsukuba (Japan)

Gediminas Račiukaitis, Center for Physical Sciences and Technology (Lithuania)

Conference Program Committee

Craig B. Arnold, Princeton University (United States)

J. Thomas Dickinson, Washington State University (United States)

Jan J. Dubowski, Université de Sherbrooke (Canada)

Bo Gu, Bos Photonics (United States)

Henry Helvajian, The Aerospace Corporation (United States)

Sami T. Hendow, ALP (United States)

Guido Hennig, Daetwyler Graphics AG (Switzerland)

Heinz P. Huber, Hochschule für Angewandte Wissenschaften München (Germany)

Michel Meunier, Ecole Polytechnique de Montréal (Canada)

Carlos Molpeceres, Universidad Politécnica de Madrid (Spain)

Yoshiki Nakata, Osaka University (Japan)

Hiroyuki Niino, National Institute of Advanced Industrial Science and Technology (Japan)

Alberto Piqué, U.S. Naval Research Laboratory (United States) 
Andrei V. Rode, The Australian National University (Australia)

Stephan Roth, BLZ Bayerisches Laserzentrum GmbH (Germany)

Klaus Sokolowski-Tinten, Universität Duisburg-Essen (Germany)

Razvan Stoian, Laboratoire Hubert Curien (France)

Koji Sugioka, RIKEN (Japan)

Xianfan Xu, Purdue University (United States)

Steven M. Yalisove, University of Michigan (United States)

\section{Session Chairs}

$1 \quad$ Industrial Applications I: Processes and Applications

Gediminas Račiukaitis, Center for Physical Sciences and Technology (Lithuania)

2 Industrial Applications II: Towards Digital Photonics Production Andreas Michalowski, Robert Bosch GmbH (Germany)

3 Diagnostics and its Applications

Guido Hennig, Daetwyler Graphics AG (Switzerland)

$4 \quad$ Thin Film and Wafer Processing

Henry Helvajian, The Aerospace Corporation (United States)

$5 \quad$ Laser Direct Writing I

Gediminas Račiukaitis, Center for Physical Sciences and Technology (Lithuania)

6 Laser Direct Writing II

Jean-Philippe Colombier, Université Jean Monnet Saint-Etienne (France)

7 Fundamental Aspects of Laser-Materials Interaction

Klaus Sokolowski-Tinten, Universität Duisburg-Essen (Germany)

8 Laser-induced Modification of Glasses or Transparent Materials

Beat Neuenschwander, Berner Fachhochschule Technik und Informatik (Switzerland)

9 3D Manufacturing in Micro- and Nanoscale

Craig B. Arnold, Princeton University (United States)

10 Laser Nanoscale Materials Processing and Manufacturing

Costas P. Grigoropoulos, University of California, Berkeley (United States) 